\title{
A internalização do conhecimento em energia solar fotovoltaica por meio da gamificação de Ambientes Virtuais de Ensino e de Aprendizagem
}

\author{
Natana Lopes Pereira, \\ Graceline de Oliveira, \\ Ramon Venson, \\ Markson Rebelo Marcolino, \\ Rangel Machado Simon, \\ Fernando José Spanhol
}

\section{INTRODUÇÃO}

O processo de ensino e de aprendizagem na Educação a Distância (EaD) vem evoluindo por meio de novas metodologias de ensino, impulsionadas pela nova forma de aprendizagem, resultante do uso das Tecnologias da Informação e Comunicação (TIC). A EaD é conceituada por Moore e Kearsley (2010) como o aprendizado que ocorre de forma planejada, onde os atores envolvidos não estão compartilhando o mesmo espaço físico, exigindo assim, técnicas especiais por meio das TIC para interação entre os usuários. Segundo Aires e Leite (2017), para maior colaboração e interação nesse processo entre aluno, professor e tutor, é necessário o incentivo ao uso de recursos tecnológicos dos Ambientes Virtuais de Ensino e de Aprendizagem (AVEA).

Os AVEA, segundo Cunha Filho, Neves e Pinto (2001 apud DIAS, 2003), são plataformas de comunicação que possibilitam ensino-aprendizagem por meio de recursos de TIC. A partir do crescimento do uso desses ambientes, e da mudança para a cultura digital, surge a necessidade de aperfeiçoar o método de ensino-aprendizagem por meio de Gestão do Conhecimento (GC). De acordo com Bassi (1999 apud BIASUTTI; EL-DEGHAIDY, 2012) a GC é um processo de criação, captura e compartilhamento de conhecimento que pode ser utilizada em duas perspectivas: por meio do uso dos recursos tecnológicos para disseminação do conhecimento e através do contexto social, podendo ocorrer o compartilhamento do conhecimento. Na busca por compreender como ocorre a criação e conversão do conhecimento, foram selecionados estudos de Takeuchi e No- 
naka (2008). Nesses estudos, os autores identificaram duas formas de conhecimento, caracterizados como tácito (vivência) e explícito (documentado), e quatro modos de conversão entre tais conhecimentos, denominados Modelo SECI - Socialização, Externalização, Combinação e Internalização.

A utilização desses modos por meio de AVEA, visando o fluxo contínuo de conversão e disseminação de conhecimento em EaD, é um processo que muitas vezes pode tornar-se complexo, não obtendo resultados positivos, devido à falta de interação entre os usuários. Assim, surge a necessidade de aplicação de métodos que incentivem a colaboração e a interação nos ambientes digitais, tornando constante ao processo de conversão do conhecimento. Uma das práticas que vem contribuindo no processo de ensino e aprendizagem é a gamificação. Segundo Klock, Cunha e Gasparini (2015), nessa tendência torna-se necessário considerar o conteúdo, forma de aprendizagem, e as atividades no ambiente.

Para Kapp (2012), o processo de gamificação pode proporcionar um ambiente agradável e divertido para o ensino, por meio de diversos incentivos como medalhas, ranking, pontuação, entre outros recursos. De acordo com esse contexto e tendo como justificativa a constante evolução das TIC aplicadas na EaD, sua flexibilidade no modo de ensino e aprendizagem, e a necessidade de um ambiente que estimule o aluno a realizar diversas atividades complexas, como é o caso do curso de sistemas fotovoltaicos (área de conhecimento engenharia), surge a seguinte questão de pesquisa:"Como utilizar os modos de conversão do conhecimento - SECI - e técnicas de Gamificação para contribuir no processo de ensino-aprendizagem em cursos de Sistemas Fotovoltaicos à distância?”

Para responder a tal questionamento, realizou-se investigação bibliográfica sobre a temática de pesquisa apresentada na segunda seção deste artigo. Os métodos utilizados para tal objetivo estão descritos na seção três. Na quarta seção, apresenta-se o modelo proposto para conversão do conhecimento e gamificação em AVEA. Na seção cinco finaliza-se este estudo com as considerações finais.

\section{FUNDAMENTAÇÃO TEÓRICA}

\subsection{GAMES E GAMIFICAÇÃO NA EDUCAÇÃO A DISTÂNCIA}

Em um contexto onde jogos podem proporcionar um ambiente agradável e divertido para o ensino, surge um processo chamado gamificação (KAPP, 2012). A gamificação consiste na reprodução de elementos de jogos, tais como filoso- 
fias, estratégias e mecânicas fora do contexto de jogos, objetivando promover o processo de ensino-aprendizagem e solução de problemas de forma motivadora (FARDO, 2013).

O processo de gamificação em cursos à distância pode colaborar para que estudantes alcancem melhores resultados com eficiência e flexibilidade, dada a capacidade desse tipo de recurso de atingir uma parcela maior de pessoas acostumadas com a nova gama de ferramentas trazidas pela era digital (SANMUGAM et al., 2016).

A gamificação pode ser implementada em uma plataforma de cursos online por meio de diferentes funções, que contribuam para despertar a percepção do estudante sobre avanço e recompensa pelas tarefas realizadas. Entre estas funções estão (STAUBITZ et al., 2017):

- Medalhas: têm a finalidade de gerar a sensação de recompensa para o término de uma ou um conjunto de tarefas importantes (ANTONACI et al., 2017; CHAUHAN; TANEJA; GOEL, 2015; SARAGURO-BRAVO; JARA-ROA; AGILA-PALACIOS, 2016; STAUBITZ et al., 2017).

- Quadro de Líderes: objetiva que uma parte dos estudantes seja motivada a buscar ranking dos melhores resultados. Porém, pode gerar efeito oposto, pela comparação entre todos os usuários que, apesar dos bons resultados, não se mantém entre os primeiros da tabela. Uma alternativa seria a inclusão de quadros sociais, para que os estudantes comparem seus resultados com outros usuários de maior identificação social (ANTONACI et al., 2017; STAUBITZ et al., 2017).

- Pontos de Experiência: são pontos recebidos pelos usuários pela conclusão de certas atividades, como a conclusão de questionários ou participação em fóruns (CHAUHAN; TANEJA; GOEL, 2015; STAUBITZ et al., 2017).

Essa lista, no entanto, não representa uma abordagem exaustiva das possibilidades a serem identificadas no processo de gamificação de todas as áreas. $\mathrm{O}$ processo de design de cursos online a distância possui a necessidade de especificação e da proposta de modelos específicos para cada área (PITEIRA; COSTA, 2017). A educação de ciências, tecnologias, engenharias e matemáticas apresentam uma grande quantidade de desafios pedagógicos na motivação dos estudantes no ensino de conceitos que são, em grande parte, abstratos (PIRKER; GUTL; ASTATKE, 2015).

Além do processo de gamificação, o uso de jogos digitais, de uma maneira geral, também pode ser um auxiliar na busca por novas ferramentas que aumentem a motivação e colaborem para o entendimento de questões de difícil com- 
preensão durante a interação com conteúdos disponibilizados em plataformas online (ZAPUŠEK; CERAR; RUGELJ, 2011).

Simões, Redondo e Vilas (2013) citam a utilização de linhas gerais para a implementação da gamificação que foram adotadas em uma plataforma online gamificada para as escolas de Portugal, considerando os seguintes apontamentos:permitir experimentações repetitivas;incluir ciclo rápidos de feedback; e aumentar a dificuldade das tarefas conforme a habilidade dos alunos.

Dividir tarefas complexas em tarefas simples e curtas permite que os alunos realizem atividades de maior complexidade sem que a motivação seja afetada. Nesse sentido, a aplicação dessas orientações em ambientes educacionais voltados à utilização de metodologias de jogos tem como objetivo proporcionar um ambiente de fácil acesso aos estudantes já familiarizados com as estratégias aplicadas em jogos digitais.

Com relação aos processos de desenvolvimento de jogos, um dos modelos mais utilizados é o MDA (Mechanics, Dynamics, Aesthetics) (HUNICKE; LEBLANC; ZUBEK, 2004), conforme descrito na seguir na Figura 1:

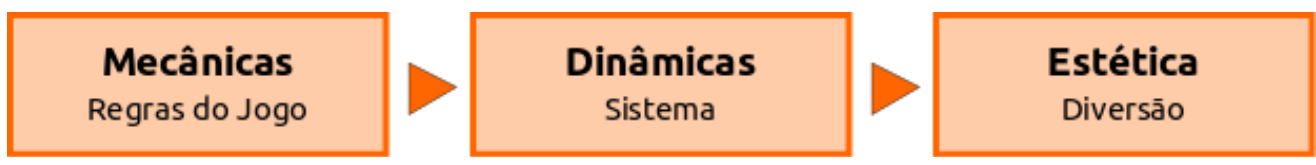

Figura 1: Modelo MDA

Fonte: HUNICKE; LEBLANC; ZUBEK (2004).

As mecânicas descrevem componentes particulares do jogo, como a representação dos dados e algoritmos. As dinâmicas descrevem o comportamento geral das mecânicas quando atuam de acordo com as entradas do usuário. Por fim, a estética caracteriza as emoções despertadas no jogador quando este interage com o sistema de jogo.

O modelo MDA não define as possibilidades relacionadas às camadas de mecânica e dinâmica do jogo, mas apresenta uma taxonomia com formas de divertimento que podem ser proporcionadas por um jogo: Sensação- jogos como forma de prazer; Fantasia- jogos como faz de conta; Narrativa - jogos como drama; Desafio - jogos como superação de obstáculos; Companheirismo - jogos como plataforma social; Descoberta - jogos como territórios desconhecidos; Expressão- jogos como autodescoberta; Submissão - jogos como passatempo. 


\subsection{A CONVERSÃO DO CONHECIMENTO EM AMBIENTES VIRTUAIS DE ENSINO E APRENDIZAGEM}

Uma questão que vem sendo estudada desde os primórdios até os dias atuais é o modo como o conhecimento é construído, e a partir deste como ele pode ser gerido e compartilhado. Segundo Maturana e Varela (2011), para que ocorra a construção e conversão do conhecimento é necessário conhecer o conhecer.

Criar o conhecimento, ou "conhecer o conhecer" não é apenas a capacidade de aprender com os outros, mas, segundo Moresi (2001 apud LACERDA et al., 2010),também é a capacidade de construir conhecimento por si só e por meio da interação entre os indivíduos. Takeuchi e Nonaka (2009) enfatizam que existem duas formas de conhecimento, sendo eles o conhecimento tácito e o conhecimento explícito. O conhecimento tácito é caracterizado por ser um conhecimento de vivência do indivíduo, pessoal, já o explícito é o conhecimento externalizado, documentado (URIARTE, 2008 apud PACHECO, 2015).

Uma pessoa pode transformar o conhecimento tácito em explícito e vice-versa, ocorrendo assim, a conversão do conhecimento. Takeuchie Nonaka (2009) defendem que a conversão do conhecimento ocorre como uma espiral, por meio da interação entre quatro (4) quadrantes de conversão do conhecimento ou, na concepção de Maturana e Varela, de conhecer o conhecer, sendo estes:

- Socialização: de tácito para tácito. A criação do conhecimento inicia com a socialização, onde o conhecimento tácito se converte em mais conhecimento tácito.

- Externalização: de tácito para explícito. A essência dessa etapa é a tradução do conhecimento tácito em formas compreensivas para o outro.

- Combinação: de explícito para explícito. A colocação do conhecimento recém-criado e do já existente proveniente de outras seções.

- Internalização: de explícito par tácito. Aprender e adquirir o novo conhecimento explícito na prática, aprender fazendo.

Assim como o modelo SECI, diversas práticas de gestão de conhecimento vêm lentamente sendo aplicadas na educação superior a distância, tendo como principal diferencial sua natureza de gerenciamento. Suas práticas, para criação e compartilhamento de conhecimento, ainda não se tornaram rotinas diárias no contexto educacional. Sua aplicação, por meio de recursos de TIC nos AVEA, pode proporcionar um ambiente colaborativo, interativo e dinâmico (BIASUTTI; EL-DEGHAIDY, 2012).

Segundo Pereira (2007), os AVEA são primordiais para a interação e cola- 
boração a distância entre os envolvidos, e sua utilização vem sendo aplicada além do ensino a distância, cada vez mais no ensino presencial. Com a evolução tecnológica, várias plataformas foram desenvolvidas e estão em constante melhoria como o MOODLE, Rodas, TelEduc, entre outros. Essas plataformas suportam diversos recursos de TIC que facilitam o gerenciamento da aprendizagem, possibilitando a administração e documentação de cursos (CAPUTI; GARRIDO, 2015).

Caputi e Garrido (2015) enfatizam que os AVEA requerem interação do aluno por meio das atividades de comunicação (e-mail, fórum e chat) e atividades de exploração (páginas online, pastas, arquivos,entre outros). Para melhor aproveitamento dos AVEA no ensino, não o tornando apenas um repositório de informações, é necessária a exploração de seus recursos, sendo fundamental não os utilizar isoladamente, mas como parte de um sistema maior que possibilite a construção de caminhos distintos para a aprendizagem do aluno (CAPUTI; GARRIDO, 2015).

\subsection{SISTEMAS DE ENERGIA SOLAR FOTOVOLTAICOS}

O curso de Energia Solar em Sistemas Fotovoltaicos, área de conhecimento da Engenharia, possui uma temática extremamente importante e complexa. Para formar profissionais capacitados, é fundamental um AVEA que suporte ou ofereça diversas atividades para melhor absorção e prática do conhecimento adquirido, uma vez que engloba saberes teóricos e técnicos, conforme descrito a seguir.

Uma das formas de energia mais utilizadas mundialmente é a energia elétrica. Cepel/Cresesb (2014) destaca que uma das opções energéticas favoráveis que possibilita a geração de energia elétrica é a solar, sendo infinita como fonte de calor e luz. Como enfatiza Teixeira (2016), a energia do sol que chega à superfície terrestre representa dez mil vezes maisa necessidade global por energia. Apenas $0,01 \%$ dessa energia seria necessária para suprir o consumo total de energia da população mundial. Essa técnica que produz energia elétrica em corrente contínua, com o uso de semicondutores no momento em que estes são iluminados por fótons existentes na radiação solar, é considerada energia solar fotovoltaica (SILVA et al., 2017).

Os menores elementos que produzem energia, as células fotovoltaicas, são compostos por um material semicondutor, especialmente pelo elemento químico mais presente na superfície terrestre, o silício (SI) (CEMIG, 2012). De acordo com o autor, a função das células fotovoltaicas é converter a potência relaciona- 
da à radiação solar em potência elétrica de corrente contínua (CC).

Como salienta Novak (2016), uma unidade composta por um grupo de células solares, conectadas eletricamente para geração de energia elétrica, é denominada módulo fotovoltaico. Nesse sentido, Cepel/Cresesb (2014) acrescenta que um grupo de 36 a 216 células fotovoltaicas interligadas em série e/ou paralelo formam um módulo fotovoltaico.

No que diz respeito aos painéis fotovoltaicos, Teixeira (2016) define que são formados por conjuntos de células fotovoltaicas interligadas entre si para converter energia solar em energia elétrica, por meio do efeito fotovoltaico, formando os sistemas de energia solar fotovoltaico. Conforme as aplicações dos sistemas fotovoltaicos, um ou mais módulos, além de dispositivos adicionais como acumuladores eletroquímicos, controladores de carga, inversores e outros materiais de proteção, formam um sistema fotovoltaico de energia (RÜTHER, 2004 apud SANTOS et al., 2017).

Os componentes básicos de um sistema fotovoltaico, segundo Cepel/Cresesb (2014), são: (1) bloco gerador que contém os módulos fotovoltaicos; (2) bloco de condicionamento de potência com os conversores, seguidor de ponta de potência máxima, inversores, controladores de carga e outros dispositivos de proteção; (3) bloco de armazenamento formado por acumuladores elétricos (baterias) e/ou outras formas de armazenamento.

A aplicação dos sistemas fotovoltaicos, como aponta Cepel/Cresesb (2014), pode ocorrer em diversas áreas com objetivos diferenciados. Entre essas aplicações estão ossistemas fotovoltaicos na área de telecomunicações e ossistemas fotovoltaicos de bombeamento de água; entre outras aplicações, como proteção catódica, cerca elétrica e dessalinização.

Para Silva, Ferreira e Benarrosh (2015), as principais vantagens dos sistemas fotovoltaicos são: ter facilidade de manutenção; poder armazenar eletricidade gerada em baterias; ajudar a reduzir a dependência por combustíveis fósseis. Com relação às desvantagens dos sistemas fotovoltaicos, o autor se refere às despesas de implementação; aos problemas relacionados à natureza da energia solar; à manutenção e substituição de baterias e impactos negativos nas etapas de produção, construção e desmatamento.

\section{METOdOLOGIA PARA CONVERSÃO DO CONHECIMENTO EM AVEA GAMIFICADOS}

Visando explicitar o problema de pesquisa (GIL, 2010) para elaboração de um método de conversão do conhecimento em AVEA gamificados no curso de 
sistemas fotovoltaicos, primeiramente realizou-se uma pesquisa exploratória. Para maior domínio da temática da pesquisa, realizou-se pesquisa bibliográfica. De acordo com Gil (2010), a pesquisa bibliográfica visa a maior aproximação com o tema abordado, é realizada por meio de análise de materiais já elaborados, como artigos científicos, livros, entre outros.

Os artigos foram relacionados de acordo as seguintes variáveis de pesquisa "AVEA" ou "AVA", "GC", "Modelo SECI", "EaD” e "Sistemas Fotovoltaicos" nas bases de dados Repositório da UFSC, Google Acadêmico e Periódicos Capes. Como critério de inclusão, foram selecionados os artigos de maior relevância que apresentassem no título, ou nas palavras-chaves, as variáveis de busca sendo posteriormente realizada análise qualitativa dos documentos selecionados. Essa abordagem, de acordo Freire (2013), objetiva explorar e descrever a complexidade de determinado problema e analisar a interação das variáveis envolvidas no estudo.

Após estudo do material selecionado, iniciou-se a proposta de estruturação do AVEA utilizando técnicas de gamificação e modos de conversão do conhecimento, conforme descrito no diagrama a seguir, representado na Figura 2:

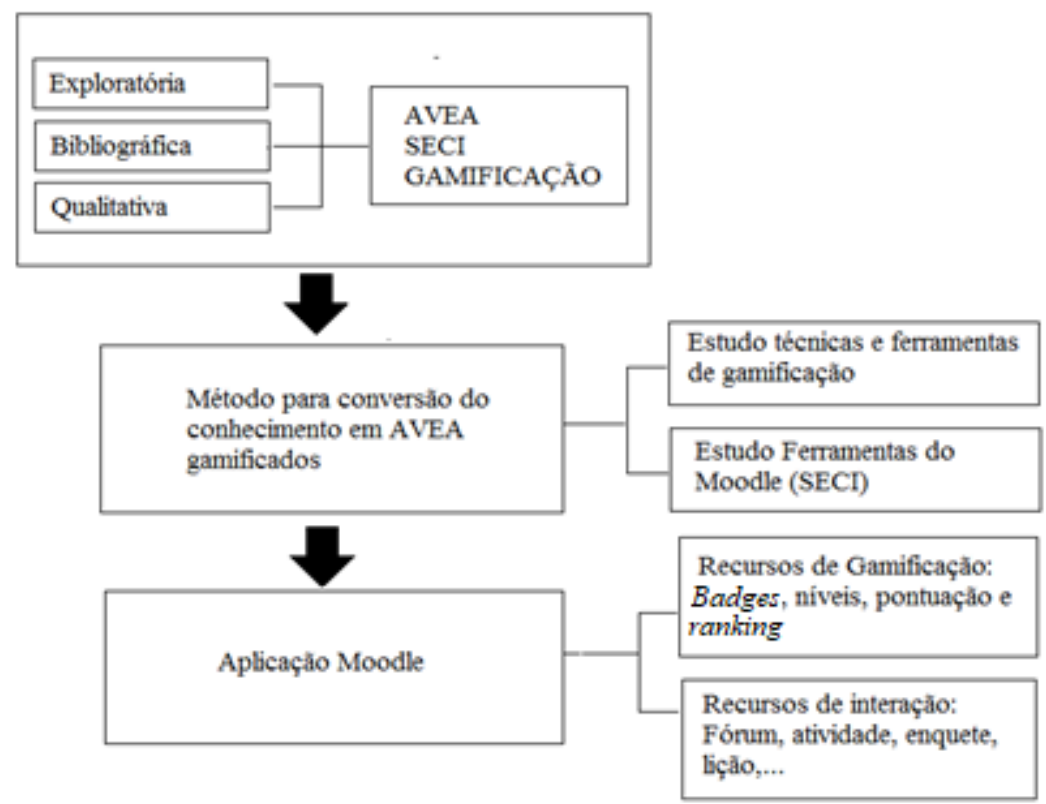

Figura 2: Diagrama estruturação da pesquisa

Fonte: Autores (2017). 
Com o intuito de elaborar um método para conversão do conhecimento em AVEA, por meio de técnicas de gamificação, utilizou-se, no roteiro inicial proposto para aplicação no ambiente, elementos de recompensa elencados por Viola (2011 apud FALCÃO; LEITE; TENÓRIO, 2014) em: classificações, níveis, pontos, medalhas, recompensas e missões. Quanto ao método de aprendizagem, utilizou-se como base, o modo de conversão do conhecimento - Modelo SECI -, aderindo como ferramenta mediadora para conversão do conhecimento os recursos de Tecnologias da Informação e Comunicação - TIC do AVEA.

O uso dos recursos de TIC das plataformas educacionais, tanto para aplicação de atividades, ou para disponibilização de materiais didáticos, por meio de diversas mídias, torna possível a internalização do conhecimento do aluno. Para que ocorra a conversão do conhecimento explícito para o tácito (última constante da espiral da conversão do conhecimento), é imprescindível estudo e utilização de forma adequada de todas as funcionalidades do AVEA. Esses ambientes segundo, Dias (2003), devem possuir características de interação intercâmbio, troca, comunicação, cooperação, colaboração, entre outros, e que possibilitem a inserção de métodos para conversão do conhecimento individual ou coletivo, o ensino e a aprendizagem.

Muitas vezes, o ambiente virtual é utilizado apenas como um repositório de informação, não sendo esta, aproveitada ou absorvida da melhor forma pelos seus usuários. Para maior eficácia na utilização desses ambientes, devem-se considerar três aspectos elencados por Biasutti e El-Deghaidy (2012): processos sociais, processos cognitivos dos usuários (externalização e internalização) e influência de ambos os processos mutuamente.

Tendo como base os processos sociais, cognitivos e de influência dos usuários no AVEA, os quadrantes de conversão do conhecimento - SECI e de gamificação (MDA) -, estruturou-se o seguinte roteiro (método) para representação e compreensão da metodologia utilizada no processo de ensino-aprendizagem, do protótipo do Curso a Distância de Sistemas Fotovoltaicos, conforme descrito no Quadro 1: 


\begin{tabular}{|l|l|l|l|}
\hline \multicolumn{1}{|c|}{ ATIVIDADES } & $\begin{array}{c}\text { CONVERSÃO } \\
\text { DO CONHECI- } \\
\text { MENTO }\end{array}$ & GAMIFICAÇÃO & $\begin{array}{l}\text { RECURSOS DO } \\
\text { AVEA }\end{array}$ \\
\hline $\begin{array}{l}\text { Conhecimento } \\
\text { prévio do tema } \\
\text { abordado }\end{array}$ & $\begin{array}{l}\text { Socialização e } \\
\text { Externalização }\end{array}$ & $\begin{array}{l}\text { Barra de progresso } \\
\text { e pontuação, níveis }\end{array}$ & $\begin{array}{l}\text { Ferramentas } \\
\text { para atividades } \\
\text { colaborativas }\end{array}$ \\
\hline $\begin{array}{l}\text { Conhecimento } \\
\text { prévio; Material } \\
\text { didático; Atividades }\end{array}$ & Combinação & $\begin{array}{l}\text { Barra de progresso } \\
\text { e pontuação, } \\
\text { níveis, medalhas }\end{array}$ & $\begin{array}{l}\text { Ferramentas para } \\
\text { disponibilização } \\
\text { de material } \\
\text { didático (recursos) } \\
\text { e Ferramentas } \\
\text { elaboração } \\
\text { de Atividades } \\
\text { individuais }\end{array}$ \\
\hline $\begin{array}{l}\text { Conhecimento } \\
\text { prévio; Material } \\
\text { didático; Atividades; } \\
\text { Construção do } \\
\text { Conhecimento }\end{array}$ & Internalização & $\begin{array}{l}\text { Barra de progresso } \\
\text { e pontuação, } \\
\text { níveis, medalhas, } \\
\text { atividades bônus }\end{array}$ & $\begin{array}{l}\text { Ferramentas } \\
\text { atividades } \\
\text { colaborativas }\end{array}$ \\
\hline
\end{tabular}

Quadro 1: Roteiro para conversão de conhecimento em AVEA Fonte:Autores (2017).

Nesse modelo, evidencia-se a importância do planejamento e do estudo do método para posterior aplicação na plataforma de ensino-aprendizagem.

\section{APLICAÇÃO DA METOdOLOGIA PROPOSTA NA PLATAFORMA MOODLE}

Após elaboração da proposta de metodologia de ensino e aprendizagem no curso de Sistemas Fotovoltaico em EaD, aplicou-se o roteiro ilustrado no quadro 1, ao AVEA MOODLE no segundo trimestre de 2017. Esse ambiente, de acordo com Aires e Leite (2017), é uma plataforma de gerenciamento de aprendizagem que oferece diversos recursos de colaboração e interação entre os alunos.

O curso, estruturado por capítulos, utilizou diversos recursos de TIC para conversão do conhecimento conforme, Figura 3. Segundo Biasutti e El-Deghaidy (2012), a criação e compartilhamento de conhecimento por meio das tecnologias no processo educacional proporcionam ao aluno a internalização do conhecimento explícito em práticas tácitas e vice-versa. 


\title{
Capítulo 5- Aplicações de Sistemas Fotovoltaicos
}

\begin{abstract}
Sistemas Fotovoltaicos Isolados; Sistemas de Bombeamento de Agua; Sistemas de telecomunicações e monitoramento remoto; Sistemas conectados à rede;
\end{abstract}

\begin{abstract}
Antes de iniciar a leitura e resolução das atividades do capítulo contribua com o fórum abaixo:
\end{abstract}

Fórum Capítulo 5

\section{Material Didático}

capitulo 5 - Aplicações de Sistemas Fotovoltaicos

FUNCIONAMENTO ENERGIA SOLAR REDE

Videoteca

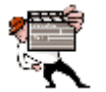

\section{Atividades}

Sistemas Conectados a Rede

Resenha Aplicação de Sistemas Fotovoltaicos

Figura 3: Estrutura MOODLE

Fonte: <MOODLE.ufsc.br>.

Para aplicação da atividade inicial, utilizou-se a ferramenta fórum, visando a socialização e externalização do conhecimento prévio sobre a temática (processos sociais e cognitivos). Em seguida, a disponibilização de material didático por meio de objetos de aprendizagem, e aplicação de atividades práticas com os recursos do MOODLE: enquete, escolha, atividade, lição. Esses recursos (Material didático e atividades), quando aplicados de forma coerente ao conteúdo abordado, podem proporcionar a combinação do conhecimento (processo cognitivo). Após a realização dessas atividades, para que ocorra a internalização do conhecimento, é necessária a aplicação de uma atividade com os recursos de comunicação colaborativa como o Fórum, Wiki ou Chat (influência de ambos os processo- cognitivos e sociais), sendo necessária a cada nova etapa, ou tópico, uma nova espiral.

Para maior motivação do aluno nesse processo, utilizaram-se técnicas de EaD e estratégias de recompensas, sendo considerados por Aires e Leite (2017) 
em sistemas gamificados. Para inserção dessas técnicas na plataforma MOODLE, foram utilizados os elementos de pontuação, níveis e ranking, tendo como mecânica e dinâmica as ações desempenhadas pelo aluno no ambiente, como realização de atividades e acesso ao material didático. A Figura 4 representa a inserção dos elementos de gamificação na plataforma MOODLE, como o ranking, nível, pontuação e progresso do aluno:

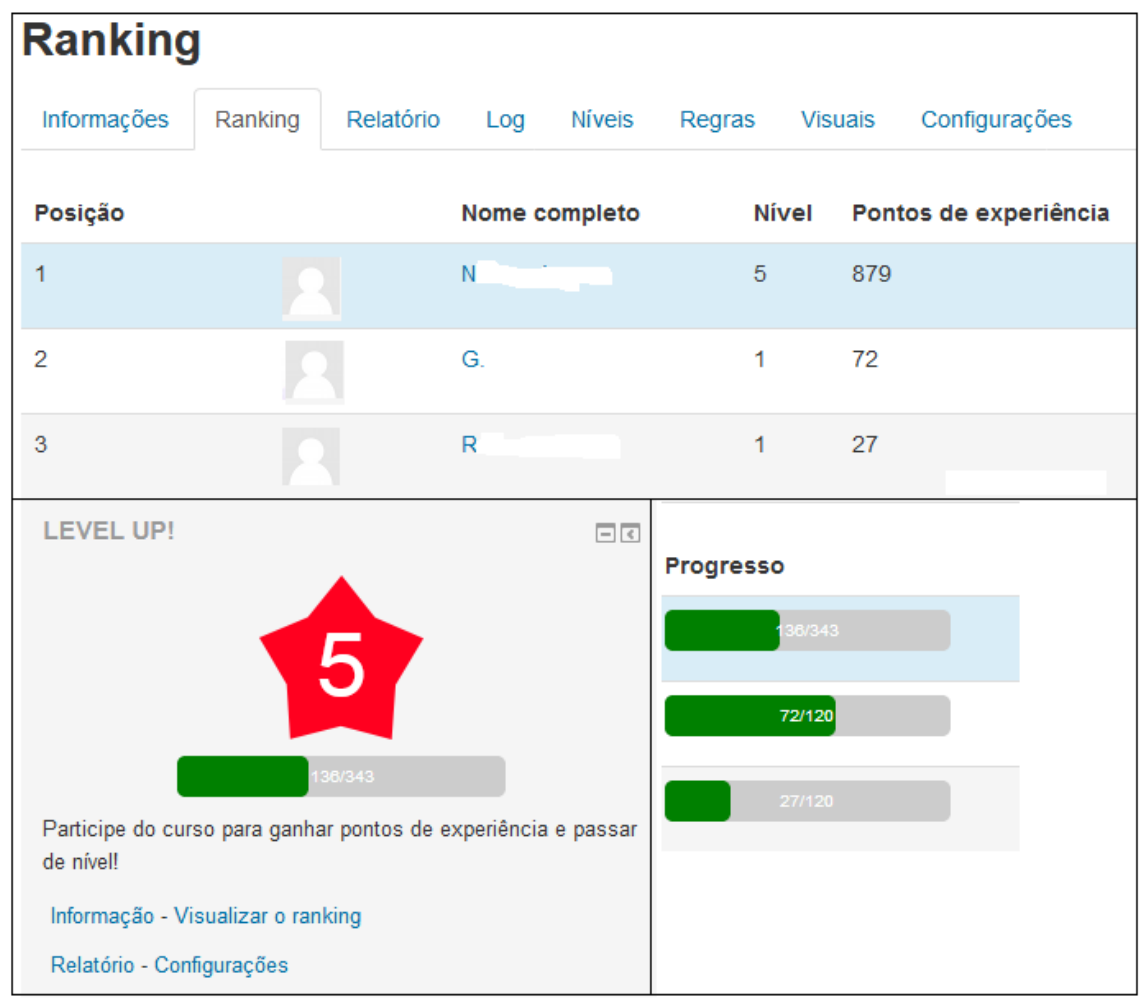

Figura 4: Recursos de gamificação

Fonte: <MOODLE.ufsc.br>.

Como estratégia para a gamificação, além dos itens já mencionados, utilizou-se no ambiente o recurso "badges", conhecidos também como "medalhas" ou "emblemas". O recurso "badges", possibilita a bonificação do aluno por meio de critérios definidos pelo professor, como, por exemplo, a conclusão de atividades, a atribuição por maior nota, a interação no ambiente, ou outras ações realizadas no MOODLE. Quando o aluno atinge o critério estabelecido pelo professor na plataforma, ele é bonificado por meio de um prêmio virtual, conforme ilustrado na Figura 5: 


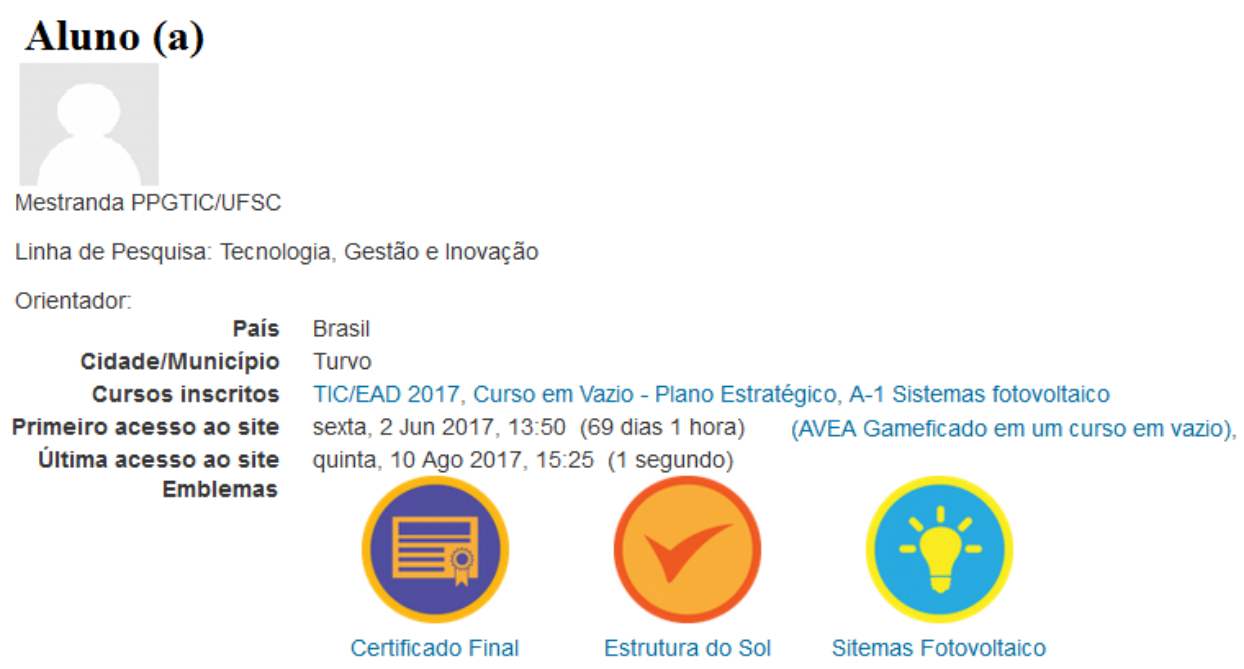

Figura 5 - Emblemas MOODLE Fonte: <MOODLE.ufsc.br>.

Com o intuito de utilizar os diversos recursos de TIC do MOODLE, e propiciar um ambiente de motivação, e maior incentivo por alta pontuação, foram utilizadas atividades condicionadas à nota. $\mathrm{O}$ aluno poderia ter acesso a um jogo digital como bônus, se atingir nota ou pontuação superior a oito (8) nas atividades propostas. A Figura 6 ilustra o jogo bônus relacionado à atividade condicionada de cada capítulo do curso:

W. Conhecendo os recursos energéticos do sol

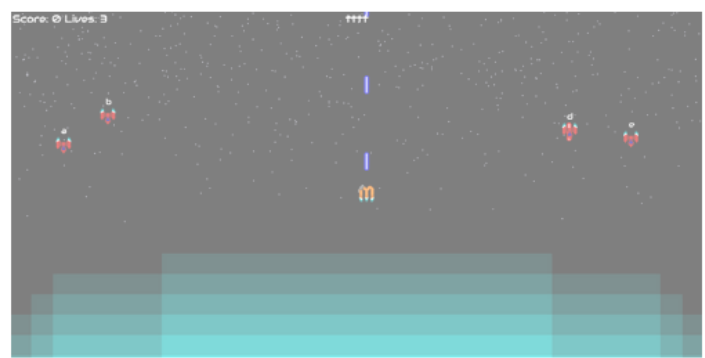

Não disponivel, a não ser que: A atividade Resenha Recurso Solar esteja marcada como concluída

Figura 6: Bônus atividade condicionada

Fonte: <MOODLE.ufsc.br>. 
Segundo Klock, Cunha e Gasparini (2015), os elementos de gamificação utilizados estão relacionados com necessidades de recompensa, de demonstrar posições, com competitividade e desafios, trazendo ao aluno a satisfação e motivação no processo de ensino e de aprendizagem. Após a configuração do ambiente, de acordo com a metodologia proposta de estudo para aplicação em cursos a distância, pode-se perceber a flexibilidade do ambiente virtual de aprendizagem MOODLE, que por meio de combinação de estratégias (gamificação e SECI), pode intensificar a participação e a motivação do aluno no processo de ensino e de aprendizagem.

\section{DISCUSSÃO DOS RESULTADOS}

Após elaboração e aplicação do método proposto ao AVEA, pode-se evidenciar que ele possui diversas ferramentas e recursos de TIC que possibilitam o ensino e a aprendizagem de acordo com o estilo de aprender da cada discente. $\mathrm{Na}$ estruturação proposta neste artigo, tendo como base recursos de gamificação, e o modo de conversão do conhecimento SECI (Socialização, Externalização, Combinação, Internalização), evidenciou-se que tal plataforma suporta o modelo descrito, uma vez que possui diversos recursos que comportam processos cíclicos para internalização do conhecimento.

Abordando os recursos de gamificação, contatou-se que o AVEA MOODLE possui ferramentas específicas, como o ranking, as medalhas e outros plugins para jogos. Além destas, há diversas possibilidades de incentivar a interação do aluno com o ambiente, adequando alguns recursos ao contexto de recompensas, dependendo do método que será abordado em aula, como, por exemplo, a aplicação de atividades condicionadas, a utilização de enquetes, tarefas entre outros.

Além do incentivo à interação por meio da gamificação, o modelo, a estrutura proposta, buscou proporcionar a mediação do processo de ensino e aprendizagem, tendo como base os modos de conversão do conhecimento SECI. A partir de estudo das ferramentas de TIC do MOODLE, pode-se evidenciar que a utilização em conjunto, de forma combinada, de tais recursos proporcionam a conversão do conhecimento de forma cíclica. Torna-se de primordial importância o planejamento prévio das dinâmicas em aula, uma vez que é necessário que as atividades propostas, e as ferramentas utilizadas estejam de acordo com o conteúdo disponibilizado, e que este seja acessível de acordo com os diversos estilos de aprendizagem. 
Assim, a partir da elaboração do modelo proposto, pode-se elencar a importância de duas variáveis: tecnologia digital e planejamento. As TIC possibilitam inúmeras maneiras de incentivar a interação entre aluno e objeto de estudo, principalmente em $\mathrm{EaD}$, mas apenas sua utilização sem um estudo detalhado sobre o método de uso e aplicação, não seriam eficientes ao processo de ensino e de aprendizagem.

\section{CONSIDERAÇÕES FINAIS}

$\mathrm{Na}$ pesquisa realizada, aplicaram-se técnicas de gamificação, tendo como base os modos de conversão do conhecimento de Takeuchi e Nonaka (2008), visando maior interação entre aluno e AVEA de um curso de sistemas fotovoltaicos em EaD. Tal aplicação visou efetivar a construção do conhecimento em cursos $\mathrm{EaD}$, por meio de práticas de gestão do conhecimento e recursos, estratégias, de gamificação. A partir da elaboração (proposta) do método para conversão do conhecimento no ambiente, e sua aplicação (protótipo) na plataforma educacional MOODLE, pode-se elencar a importância do planejamento, e do domínio de seus recursos de TIC. Falcão e Tenório (2014) enfatizam a importância do correto incentivo aos alunos, por meio das ferramentas dos AVEA, visando a um ambiente mais interativo e colaborativo.

Evidencia-se assim, a estratégia utilizada em relacionar o estudo do método por meio de gestão do conhecimento (modelo SECI) e técnicas de incentivo e motivação por meio da gamificação. A partir desse planejamento, obteve-se como produto final um ambiente propício a constante conversão das informações disponibilizadas e compartilhadas, em conhecimento no processo de EaD. Segundo Klock, Cunha e Gaparini (2017), a gamificação possibilita que o aluno aprenda se divertindo, tornando o processo de ensino e de aprendizagem motivador, sendo necessário o método adequado de acordo com o usuário.

Destaca-se assim, a importância e necessidade de estudar formas eficientes, novos métodos de ensino aprendizagem em EaD por meio dos AVEA. Aplicar a gestão do conhecimento em tais plataformas, tornando o compartilhamento, a disseminação, e a conversão do conhecimento uma rotina diariamente incentivada e alimentada é o principal diferencial para sucesso dos cursos a distância (BIASUTTI; EL-DEGHAIDY, 2012).

Enfatiza-se que o estudo apresentou a estrutura para um ambiente que possibilite a conversão do conhecimento em cursos de Sistemas Fotovoltaicos, utilizando como estratégia métodos de gamificação. A partir da elaboração do protótipo, é identificada a necessidade de sua aplicação, de acordo com o perfil dos usuários, para maior efetividade e validação da pesquisa realizada. 


\section{REFERÊNCIAS}

AIRES, S. F; LEITE, J. C. Estudo e implementação de técnicas de motivação no MOODLE. In: CONGRESSO DA SOCIEDADE BRASILEIRA DE COMPUTAÇÃO, 37., 2017, São Paulo. Anais. São Paulo: Universidade Presbiteriana Mackenzie, 2017. 15 p. Disponível em: <http://csbc2017.mackenzie.br/public/files/14-sbsc/completos/13. pdf>. Acesso em: 5 ago. 2017.

ANTONACI, A. et al. Gamification in MOOCs to enhance users' goal achievement. In: 2017 IEEE GLOBAL ENGINEERING EDUCATION CONFERENCE (EDUCON). 2017, [S.1.] .Anais... [S.1.]: [s.n.], 2017. p. 1654-1662.

BIASUTTI, M.; EL-DEGHAIDY, H. Using Wiki in teacher education: impact on knowledge management processes and student satisfaction. Computers \& Education, [S.l.], v. 59, n. 3, p. 861-872, nov. 2012. Elsevier BV.

CAPUTI, V.; GARRIDO, A. Student-oriented planning of e-learning contents for MOODLE. Journal Of Network And Computer Applications, [S.1.], v. 53, p. 115-127, jul. 2015. Elsevier BV.

CEMIG. Alternativas energéticas: uma visão. Belo Horizonte: Cemig, 2012. 373 p.

CEPEL/CRESESB. Manual de engenharia para sistemas fotovoltaicos. Rio de Janeiro, RJ: Cepel-Cresesb, 2014. 530 p.

CHAUHAN, J.; TANEJA, S.; GOEL, A. Enhancing MOOC with augmented reality, adaptive learning and gamification. In: 2015 IEEE INTERNATIONAL CONFERENCE ON MOOCS, INNOVATION AND TECHNOLOGY IN EDUCATION (MITE), 3.,2015, [S.1.]. Anais... [S.1.]: [s.n.], 2015. p. 348-353.

DIAS, R. F. Ambientes virtuais de aprendizagem - uma metodologia para avaliação de software. 2003. 151 f. Dissertação (Mestrado) - Curso de Engenharia de Produção, Universidade Federal de Santa Catarina, Florianópolis, 2003.

FALCÃO, A. P.; LEITE, M. D.; TENÓRIO, M. M. Ferramenta de apoio ao ensino presencial utilizando gamificação e design de jogos. 2014. Disponível em: <http://www. br-ie.org/pub/index.php/sbie/article/view/2981/2492>. Acesso em: 8 ago. 2017.

FARDO, M. L. A gamificação aplicada em ambientes de aprendizagem. RENOTE, v. 11, n. 1, 5 ago. 2013. Disponível em: <http://seer.ufrgs.br/index.php/renote/article/ view/41629>. Acesso em: 31 jul. 2017.

FREIRE, P. S. Aumente qualidade e quantidade de suas publicações científicas: manual para elaboração de projetos e artigos científicos. Curitiba, PR: CRV, 2013.

GIL, A. C. Como elaborar projetos de pesquisa. São Paulo: Atlas, 2010.

HUNICKE, R.; LEBLANC, M.; ZUBEK, R. MDA: A formal approach to game design and game research. [S.1.]: [s.n.], 2004. v. 4.p. 1722.

KAPP, K. M. The gamification of learning and instruction: game-based methods and strategies for training and education. San Francisco, CA: Pfeiffer, 2012.

KLOCK, A. C. T.; CUNHA, L. F.; GASPARINI, I. Um modelo conceitual para a gamificação de Ambientes Virtuais de Aprendizagem. Cinted: Novas Tecnologias na Edu- 
cação, Joinville, v. 13, n. 1, p.1-10, jul. 2015.

LACERDA, M. R. M. et al. Criação e compartilhamento de conhecimento em ambientes virtuais de ensino-aprendizagem. Renote - Revista Novas Tecnologias na Educação, Porto Alegre, v. 1, n. 8, p.1-10, jul. 2010.

MATURANA, H. R.; VARELA, F. J. A árvore do conhecimento:as bases biológicas da compreensão humana. 9. ed. São Paulo: Palas Athena, 2011. 288 p.

MOORE, M. G.; KEARSLEY, G. Educação a Distância: uma visão integrada. São Paulo: Cengace Learning, 2010. 398 p.

NOVAK, M. V. Análise de modelos matemáticos de temperatura de módulos fotovoltaicos e avaliação energética a partir de dados da casa solar eficiente. $2016.55 \mathrm{f}$. TCC (Graduação) - Curso de Engenharia de Energia, Universidade Federal de Santa Catarina - UFSC, Araranguá, 2016.

PACHECO, D. C. Maturidade em gestão do conhecimento da prefeitura municipal de criciúma: o caso dos setores de convênios. 2015. 190 f. Dissertação (Mestrado) Curso de Gestão do Conhecimento, Universidade Federal de Santa Catarina, Florianópolis, 2015.

PEREIRA, A. T. C. Ambientes Virtuais de Aprendizagem: em diferentes contextos. Rio de Janeiro: Ciência Moderna Ltda, 2007. 210 p.

PIRKER, J.; GUTL, C.; ASTATKE, Y.Enhancing online and mobile experimentation using gamification strategies. In: 2015 3RD EXPERIMENT INTERNATIONAL CONFERENCE (EXP.AT'15),3., 2015, [S.1.] . Anais... [S.1.]: [s.n.], 2015. p. 224-229.

PITEIRA, M.; COSTA, C. J. Gamification: conceptual framework to online courses of learning computer programming. In: 2017 12TH IBERIAN CONFERENCE ON INFORMATION SYSTEMS AND TECHNOLOGIES (CISTI), 12.,2017,[S.1.]. Anais... [S.1.]: [s.n.], 2017. p. 1-7.

SANMUGAM, M. et al. The impacts of infusing game elements and gamification in learning. In: 2016 IEEE 8TH INTERNATIONAL CONFERENCE ON ENGINEERING EDUCATION (ICEED), 8.,2016, [S.1.]. Anais... [S.l.]: [s.n.], 2016. p. 131-136.

SANTOS, D. R. C. G. et al. Energia solar fotovoltaica: um estudo de caso da aplicação no sistema de iluminação em uma instituição de ensino profissionalizante. Revista Gestão Inovação e Tecnologias, [S.1.], v. 7, n. 2, p.3859-3875, 26 jun. 2017. Associação Acadêmica de Propriedade Intelectual.

SARAGURO-BRAVO, R. A.; JARA-ROA, D. I.; AGILA-PALACIOS, M. Techno-instructional application in a MOOC designed with gamification techniques. In: 2016 INTERNATIONAL CONFERENCE ON EDEMOCRACY EGOVERNMENT (ICEDEG),3., 2016, [S.1.]. Anais... [S.1.]: [s.n.], 2016. p. 176-179.

SILVA, B. F.; FERREIRA, J. S.; BENARROSH, P. F. P. M. Energia solar: benefícios das placas solares em telhas de barro sustentáveis. R. Farociência, Porto Velho, v. 2, n. 2, p.128-131, dez. 2015. Disponível em: <http://www.faro.edu.br/farociencia/index. php/FAROCIENCIA/article/view/80>. Acesso em: 6 ago. 2017.

SILVA, J. A.et al. Energia solar fotovoltaica: um tema gerador para o aprendizado de 
Física. Scientia Plena,[S.1.], v. 13, n. 1, p.1-8, 13 jan. 2017. Associação Sergipana de Ciência.

SIMÕES, J.; REDONDO, R. D.; VILAS, A. F. A social gamification framework for a K-6 learning platform. Computers in Human Behavior, Advanced Human-Computer Interaction, v. 29, n. 2, p. 345-353,1 mar. 2013.

STAUBITZ, T. et al. The gamification of a MOOC platform. In: 2017 IEEE GLOBAL ENGINEERING EDUCATION CONFERENCE (EDUCON), 2017, [S.1.]. Anais... [S.l.]: [s.n.], 2017. p. 883-892.

TAKEUCHI, H.; NONAKA, I. Gestão do conhecimento. Porto Alegre: Bookman, 2008.

TEIXEIRA, C. N. C. Incorporação de sistemas solares fotovoltaicos numa indústria agroalimentar. 2016. 104 f. Dissertação (Mestrado) - Curso de Engenharia Eletrotécnica e de Computadores, Universidade de Trás-os-Montes e Alto Douro, Vila Rela, 2016.

ZAPUŠEK, M.; CERAR, Š.; RUGELJ, J. Serious computer games as instructional technology. In: 2011 PROCEEDINGS OF THE 34TH INTERNATIONAL CONVENTION MIPRO,34., 2011, [S.1.] . Anais... [S.1.]: [s.n.], 2011. p. 1056-1058. 